\title{
Time and resources of peripherally inserted central catheter insertion procedures: a comparison between blind insertion/chest X-ray and a real time tip navigation and confirmation system
}

\author{
This article was published in the following Dove Press journal: \\ ClinicoEconomics and Outcomes Research \\ 7 February 2017 \\ Number of times this article has been viewed
}

\section{Kenneth J Tomaszewski \\ Nicole Ferko ${ }^{2}$ \\ Sarah S Hollmann ${ }^{2}$ \\ Simona C Eng ${ }^{3}$ \\ Howard M Richard ${ }^{4}$ \\ Lynn Rowe ${ }^{5}$ \\ Susan Sproule ${ }^{6}$}

'KJT Group, Inc., Honeoye Falls, NY USA; ${ }^{2}$ Cornerstone Research Group, Inc., Burlington, ON, Canada; ${ }^{3}$ Department of Hospitalist/Inpatient Services, Peninsula Regional Medical Center, Salisbury, MD, USA; ${ }^{4}$ Department of Diagnostic Radiology, University of Maryland Medical Center, Baltimore, MD, USA; ${ }^{5}$ Department of Research, Florida Hospital, Maitland, FL, USA; 'Department of Diagnostic Imaging, Unity Hospital, Rochester, NY, USA

\section{Video abstract}

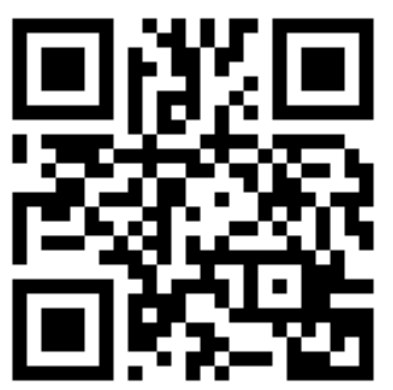

Point your SmartPhone at the code above. If you have a $Q R$ code reader the video abstract will appear. Or use: http://youtu.be/FrGLuFP_dOA

Correspondence: Kenneth J Tomaszewski KJT Group, Inc., 6 East Street, Honeoye Falls, NY 14472, USA

Tel +l 5856248050

Email ken@kjtgroup.com
Background: The Sherlock 3CG ${ }^{\mathrm{TM}}$ Tip Confirmation System (TCS) provides real-time peripherally inserted central catheter (PICC) tip insertion information using passive magnetic navigation and patient cardiac electrical activity. It is an alternative tip confirmation method to fluoroscopy or chest X-ray for PICC tip insertion confirmation in adults. The purpose of this study was to evaluate time and cost of the Sherlock 3CG TCS and blind insertion with chest $\mathrm{X}$-ray tip confirmation (BI/CXR) for PICC insertions.

Methods: A cross-sectional, observational Time and Motion study was conducted. Data were collected at four hospitals in the US. Two hospitals used Sherlock 3CG TCS and two hospitals used BI/CXR to place/confirm successful PICC tip location. Researchers observed PICC insertions, collecting data from the beginning (ie, PICC kit opening) to catheter tip confirmation (ie, released for intravenous [IV] therapy). An economic model was developed to project outcomes for a larger population.

Results: A total of 120 subjects were enrolled, with 60 subjects enrolled in each arm and 30 enrolled at each of the four US hospitals. The mean time from initiation of the PICC procedure to the time to release for IV therapy was 33.93 minutes in the Sherlock 3CG arm and $176.32 \mathrm{~min}-$ utes in the BI/CXR arm $(p<0.001)$. No malpositions were observed for PICC insertions using the Sherlock 3 CG TCS, while $20 \%$ of subjects in the BI/CXR arm had a malposition. BI/CXR subjects had significantly more total malpositions (mean 0.23 vs. $0, p<0.001$ ). For a hypothetical population of 1,000 annual patients, adoption of Sherlock 3CG TCS was predicted to be cost saving compared with BI/CXR in all three analysis years.

Conclusion: The results from this study demonstrate that Sherlock 3CG TCS, when compared with $\mathrm{BI} / \mathrm{CXR}$, is a superior alternative with regard to time to release subject to therapy, malposition rates, and minimization of $\mathrm{X}$-ray exposure.

Keywords: vascular access devices, catheters, catheterization, peripheral, X-ray therapy, cost analysis, Time and Motion studies

\section{Introduction}

Vascular access products include peripheral and central catheters, which deliver intravenous (IV) therapy into the bloodstream. Peripherally inserted central catheters (PICCs) are central catheters inserted peripherally (usually in the brachium) with the tip of the catheter residing just above the right atrium. Compared with other types of central venous access, there are several key advantages in using PICCs, including low complication rates, easy access, and low cost. ${ }^{1}$ 
Bedside PICC insertion consists of threading the catheter from the selected venous access site into the peripheral deep vein of the upper arm through the central venous system until the distal tip of the device resides in the lower third of the superior vena cava (SVC) near the cavoatrial junction..$^{2-5}$ Without the use of bedside navigation technology, this is often referred to as "blind insertion". After insertion, anatomical catheter tip confirmation is required prior to initiation of IV therapy. Confirmation is completed with a chest X-ray (CXR). Radiology personnel either transport portable CXR equipment to the bedside and/or transport the patient to the medical imaging department. Interpretation of the CXR is completed by an on-site or remote diagnostic radiologist.

There are several limitations of inserting PICCs with blind insertion with confirmatory CXR (BI/CXR). Without real-time tip navigation and confirmation, incorrect PICC tip location (malposition) with bedside insertions frequently occurs. ${ }^{6,7}$ Malpositions have been shown to lead to increased risk of cardiac arrhythmias, ${ }^{8-15}$ venous thrombosis, ${ }^{79-11,14-17}$ cardiac tamponade, ${ }^{9,12-14,18}$ vessel perforation, ${ }^{10,15}$ and catheter malfunction. . $^{7-11,14,15,17,19}$

Prior to the year 2000, the vast majority of PICCs were placed by interventional radiology physicians using fluoroscopy for visual guidance of the catheter tip and confirmation of proper final location. Important advances have been made in bedside PICC line insertion navigation and confirmation to allow a skilled nurse to complete the procedure vs. relying on interventional radiology (IR) physicians. For PICC insertion, limiting the reliance on IR may reduce patient wait times, ${ }^{20,21}$ delays in the initiation of therapy,${ }^{20}$ difficulties and risks with transport of critically ill/intensive care unit patients to the IR suite, ${ }^{22,23}$ and radiation exposure to patients and staff. ${ }^{10,11,22,23}$ Bedside PICC insertion has also been shown to reduce hospital operational costs by $\sim 65 \%$ compared with PICC insertions performed in IR. ${ }^{20,24}$

Introduced in 2011, the Sherlock 3CG ${ }^{\mathrm{TM}}$ Tip Confirmation System (TCS) (Bard Access Systems, Salt Lake City, UT, USA) is indicated for guidance and positioning of PICCs. It provides real-time PICC tip location information by using passive magnet navigation to the proximity of a pre-positioned sensor on the patient's chest. When inserting PICCs, providers place a sensor on a patient's chest before sterilizing the insertion site. Two electrocardiogram (ECG) electrodes are placed on the patient's torso. A specific catheter is used for insertion that includes an intravascular electrode within the catheter lumen. Using these electrodes, the system gathers the patient's cardiac electrical activity to confirm the tip location in relation to the patient's sinoatrial (SA) node near the cavoatrial junction. Using the Sherlock 3CG display monitor, providers see real-time tip location to aid in correct tip placement.

In patients with a distinct $\mathrm{P}$-wave in their ECG signal, the Sherlock 3CG TCS is indicated for use as an alternative method to CXR and fluoroscopy for PICC tip insertion confirmation in adult patients. Limiting but not contraindicated situations for this technique are in patients where alterations of cardiac rhythm change the presentation of the P-wave as in atrial fibrillation, atrial flutter, severe tachycardia, and pacemaker-driven rhythm. In such patients, who are easily identifiable prior to catheter insertion, the use of an additional method is required to confirm PICC tip location. ${ }^{25}$

Studies have shown the Sherlock tip location technology and the Sherlock 3CG TCS system have low rates of malpositions (effectiveness); ${ }^{11,23,26,27}$ however, there is limited real-world evidence as to its effect in reducing costs or creating efficiency in the insertion and tip confirmation process. It is important to fully detail the differences in time and costs. To accomplish this, C. R. Bard, Inc. commissioned a Time and Motion study.

An understanding of such differences is needed for health care decision-makers (clinical and financial) in order to make well-informed choices regarding alternatives to the $\mathrm{BI} / \mathrm{CXR}$ process. The aim of this study was to evaluate time and resource use differences associated with the Sherlock 3CG TCS and the BI/CXR process from real-world experiences and project the results for a larger US population. Incorporating real-world data into an economic model would allow for the examination of the potential impact of annualized cost savings in a single institution with a larger patient population.

\section{Methods}

\section{Study setting and design}

This study was a cross-sectional, observational design, characterized as a Time and Motion study. Time and motion have been shown to be more accurate than self-report activity time. ${ }^{28}$ The Suggested Time and Motion Procedures (STAMP) ${ }^{29}$ checklist was considered and used as a guideline for overall protocol and analytic planning. We compared two approaches for PICC line insertion and subsequent confirmation of the catheter's tip location, the Sherlock 3CG TCS and $\mathrm{BI} / \mathrm{CXR}$, both at the bedside. The two approaches are currently in use and as such are not investigational. There was one pre-specified null hypothesis for this study: the mean time from the initiation of PICC line insertion until the subject is cleared for therapy using the Sherlock 3CG TCS is the same as when using BI/CXR. 
Subjects were enrolled at two US institutions in each arm (four total sites). Two sites used the Sherlock 3CG TCS and two sites used BI/CXR as their primary method of PICC line insertion. Each of the four investigative sites received Institutional Review Board approval from the Western Institutional Review Board (WIRB), the Rochester Regional Health System's (RRHS) Clinical Investigation Committee (CIC), or the University of Maryland School Institutional Review Board.

Site health care providers identified potential subjects referred for a PICC insertion as part of their routine care (ie, based on their hospital's standard protocol). Subjects were not randomized to each arm; instead, they were enrolled sequentially in each arm based on the technology used at their site. Subjects met all eligibility criteria provided in Table 1. Subjects provided written informed consent before they were enrolled in the study.

On-site study personnel observed clinicians placing and confirming PICC lines and conducted brief interviews with clinicians and facility management. Paper-based case

Table I Subject inclusion and exclusion criteria

\section{Inclusion criteria}

I. Indicated for a PICC based on institutional practices.

2. Able to read and comprehend English and has signed the ICF to participate in the study.

3. PICC line is placed while a study observer is available and on the study site at the time of placement.

\section{Exclusion criteria}

I. Infection, bacteremia, or septicemia is known or suspected.

2. Body size is insufficient to accommodate the size of the implanted device.

3. Known or is suspected to be allergic to materials contained in the device. Materials in the device include polyurethane, stainless steel, polyimide, silicone, PTFE, and nickel titanium.

4. Past irradiation of prospective insertion site.

5. Previous episodes of venous thrombosis or vascular surgical procedures at the prospective placement site.

6. Local tissue factors will prevent proper device stabilization and/or access.

7. Under the age of 22 years.

8. Receiving the PICC as a replacement with an over-the-wire exchange.

9. Pregnant or lactating.

10. Anatomical irregularities (structural and vascular of the central venous system) that may compromise catheter insertion in both the primary arm and the contralateral arm.

II. Previously enrolled in this clinical study or is participating in another clinical study that is contraindicative to the treatment or outcomes of this investigation.

12. Artificial heart or heart transplant.

13. Anatomical abnormalities of the central venous system.

14. Atrial fibrillation or other atrial arrhythmias in which a P-wave was not consistently present on ECG.

15. Clinician is unable to obtain accurate external measurement due to anatomical abnormalities or personal/medical equipment.

Abbreviations: PICC, peripherally inserted central catheter; ICF, informed consent form; PTFE, polytetraflourine; ECG, electrocardiogram. report forms (CRFs) were used to collect data. Data were transcribed into an electronic database using Stata (version 14.1, StataCorp).

Subjects were observed and outcomes recorded from the beginning of a PICC insertion procedure (ie, opening of the PICC insertion procedural kit) until the subject was released for the administration of their given therapy. A limited subject follow-up consisted of a review of the subject's electronic medical records (EMRs) related to the PICC line insertion procedure. Study observers followed the nurse(s) to the potential subject's room. Subjects were followed only up to the point at which the PICC tip location was confirmed, and they were released to receive IV therapy. Once they were released to receive therapy, and review of EMR data was complete, there was no other subject follow-up.

\section{Data collection}

This study was designed with one primary end point and eight secondary end points, shown in Table 2.

In addition to these end points, a detailed cost analysis was conducted. Data related to non-physician labor, IR national average payment (Medicare), CXR cost, initial insertion materials, and additional materials to correct malpositions were collected to be used as inputs to the cost analysis.

Additional notable time points were calculated to explain differences in overall procedural time. Nurse time associated with a malposition was defined as the time between when the nurse opened gathered materials for correcting the malposition (eg, PICC kit, dressing change kit, saline, syringe) and when the subject was released for IV therapy. The subject time in IR was based on the time the subject arrived in IR and when they were released for IV therapy. Study observers

Table 2 Study end points

\section{Primary end point}

I. Time from initiation of procedure (opening of PICC kit) to release for IV therapy.

\section{Secondary end points}

I. Total number of CXRs performed per subject.

2. Number of malpositions.

3. Procedures and materials needed to correct malpositions.

4. HCP procedural satisfaction.

5. Number of additional VADs required due to PICC not being ready for use.

6. Number of missed doses due to PICC not being ready for use.

7. Number of missed laboratory draws due to PICC not being ready for use.

8. Number of overtime hours worked per placement.

Abbreviations: PICC, peripherally inserted central catheter; IV, intravenous; CXRs, chest X-rays; HCP, health care professional; VADs, vascular access devices. 
did not follow subjects to IR; as a result, this time includes both the procedural time and the waiting time for the subject.

\section{Statistical analysis}

Sample size calculations were performed to determine the appropriate number of cases in each group based on testing whether the difference, if any, in the mean time from the initiation of PICC line insertion until the subject was cleared for therapy is statistically significant. Calculations were conducted using a significance level of two-sided $\alpha 0.05$ and power of $80 \%$. Based on expected differences in observed procedural and confirmation times, a total sample size of 120 subjects was selected with two study arms using Satterthwaite's formula (nQuery 7.0), with 60 subjects per arm.

A summary of statistical methods for study end points is shown in Table 3 . Tests of $\alpha<0.05$ were considered statistically significant. Tests for normality using a Shapiro-Wilk test were used to determine whether a two-sample $t$-test assuming unequal variances or a Wilcoxon rank sum test was appropriate. Three analyses were pre-specified. Additional post hoc analyses were performed but were not pre-specified and not statistically controlled for possibly inflating falsepositive error and therefore can only be considered as exploratory or hypothesis-generating but not confirmatory evidence.

Costs were calculated based on site-provided cost data and recorded resource usage. Material cost was calculated as the purchase cost of each PICC kit, additional insertion materials, and materials required for malposition adjustments. CXR cost per PICC insertion was calculated as the

Table 3 Statistical analyses

\begin{tabular}{|c|c|c|}
\hline End points & Statistical analysis & $\begin{array}{l}\text { Pre- } \\
\text { specified }\end{array}$ \\
\hline $\begin{array}{l}\text { Time elapsed from the beginning } \\
\text { of the PICC insertion procedure } \\
\text { to catheter tip confirmation } \\
\text { (subject released for IV therapy) }\end{array}$ & Wilcoxon rank sum test & Yes \\
\hline Number of CXRs & Wilcoxon rank sum test & Yes \\
\hline $\begin{array}{l}\text { Number of subjects with } \\
\text { malpositions during the initial } \\
\text { PICC insertion }\end{array}$ & $\begin{array}{l}\text { Two-sample test of } \\
\text { proportions }\end{array}$ & Yes \\
\hline Overall procedural satisfaction & Wilcoxon rank sum test & No \\
\hline $\begin{array}{l}\text { Number of total missed } \\
\text { medication doses per subject }\end{array}$ & Wilcoxon rank sum test & No \\
\hline $\begin{array}{l}\text { Number of total missed } \\
\text { laboratory draws per subject }\end{array}$ & $\begin{array}{l}\text { Two-sample } t \text {-test } \\
\text { assuming unequal } \\
\text { variances }\end{array}$ & No \\
\hline $\begin{array}{l}\text { Number of overtime hours } \\
\text { worked per subject }\end{array}$ & $\begin{array}{l}\text { Two-sample } t \text {-test } \\
\text { assuming unequal } \\
\text { variances }\end{array}$ & No \\
\hline Procedural costs & Wilcoxon rank sum test & No \\
\hline
\end{tabular}

Abbreviations: PICC, peripherally inserted central catheter; IV, intravenous; CXRs, chest X-rays. reported operational cost to perform a CXR. Non-IR labor cost was calculated by determining the staff and hourly rates for those inserting/assisting with the procedure. IR cost was determined using the 2016 Medicare national payment for repositioning a PICC under fluoroscopy (CPT 36597) for outpatient hospital as a conservative estimate, given IR data collection was limited.

\section{Modeled economic analysis}

A health economic model was developed using Excel (version 2013, Microsoft) to project outcomes for Sherlock 3CG TCS compared with BI/CXR over 3 years for a hypothetical population of 1,000 annual patients from a hospital decision-maker perspective. The size of the hypothetical population was assumed to represent a typical US hospital. The model input data were primarily derived from actual facility experiences observed in the Time and Motion study (eg, resource utilization associated with initial PICC insertion and malposition adjustment). Specific resource parameters included the CXRs required (eg, for patients requiring initial CXRs, CXRs after malposition, and additional CXRs without adjustment), the materials, and personnel required to correct malpositions (eg, patients requiring treatment at the bedside, in the radiology unit, or no treatment). Unit costs were primarily obtained from the Time and Motion study or from published sources representing national averages based on 2016 Centers for Medicare \& Medicaid Services (CMS) reports. Capital equipment costs were included in year one of the cost analysis.

The model assumed a $100 \%$ switch from BI/CXR to the Sherlock 3CG TCS for all eligible patients in the hypothetical US hospital. The cost projections included 1) the total cost per PICC placement method per year, 2) the incremental difference in cost between the two PICC placement methods per year, and 3 ) the cumulative change (ie, incremental difference) in cost over the 3-year time horizon. The clinical projections included the incremental difference between $\mathrm{BI} /$ CXR and Sherlock 3CG TCS for 1) CXRs, 2) nurse overtime, and 3 ) patient wait time for radiology malposition correction. A schematic of the analytic design, input parameters, and projected cost and clinical outcomes is presented in Figure 1.

Given potential uncertainty in model parameters, sensitivity analyses may be conducted to test the impact of each parameter on model outcomes; therefore, a one-way sensitivity analysis was performed on all resource and cost inputs to identify the most impactful parameters on the model results. Resource and cost inputs were varied individually by adjusting base case values by $\pm 20 \%$ to produce low and high estimates. 
Design

Perspective

U.S. hospital decision-maker

Population

Hypothetical cohort of 1,000 patients

Time horizon

3 years

PICC placement methods

- BI/CXR (blind insertion + chest X-ray tip confirmation)

- Sherlock 3CG TM TCS

Cost analysis

$100 \% \mathrm{BI} / \mathrm{CXR}$ vs

$100 \%$ Sherlock 3CG ${ }^{\text {TM }}$ TCS

Inputs parameters

Outcomes

All inputs were derived from the time and motion study unless indicated $\left({ }^{*}\right)$

\section{Resource inputs}

- Initial chest X-rays

- Additional chest X-rays after malposition adjustment

- Chest X-rays but no adjustment

- Malposition adjustment distribution

- Bedside staff time for malposition adjustment

- Patient wait time for IR malposition correction ${ }^{\#}$

- Nurse over time
Cost inputs

- Average upcharge cost per PICC for Sherlock 3CG ${ }^{\mathrm{TM}}$ TCS vs. BI/CXR

- Capital equipment costs for each PICC placement method $^{*}$

- Chest X-ray cost ${ }^{\star}$

- Material costs to correct malposition at bedside

- Bedside staff wage

- Cost of procedure requiring IR to correct malpositions*
Cost projections

- Total costs per year for each PICC placement method

- Incremental difference in cost per year

- Cumulative change in cost over 3 years

\section{Clinical projections} Incremental difference in:

- Chest X-rays

- Patient wait time for IR malposition correction"

- Nurse overtime

Figure I Analytic framework.

Notes: *Unit costs that were not derived from the Time and Motion study. "Data from the Time and Motion study that were utilized for clinical projections only (ie, not included in the cost analysis).

Abbreviations: PICC, peripherally inserted central catheter; TCS, Tip Confirmation System; BI/CXR, blind insertion with confirmatory chest X-ray; IR, Interventional Radiology.

\section{Results}

One hundred and twenty-eight subjects were enrolled across both arms. A total of 120 subjects completed the study and are included in the analysis. Eight enrolled subjects discontinued as screen failures. These subjects initially met subject eligibility for all inclusion/exclusion requirements but subsequently met insertion procedure exclusion criteria after new information was obtained after enrollment.

The mean time from initiation of the PICC procedure to the time when the subject was released for IV therapy was 33.93 minutes in the Sherlock 3CG arm and 176.32 minutes in the BI/CXR arm (Table 4). The Wilcoxon rank sum test indicated that the Sherlock 3CG TCS has a statistically significant different procedure time than BI/CXR with the mean procedure time for the Sherlock 3CG TCS arm being less than the mean procedure time for the $\mathrm{BI} / \mathrm{CXR}$ arm $(p$ $<0.001$ ).

Nurse time associated with a malposition was defined as the time interval when the nurse opened gathered materials for correcting the malposition (eg, PICC kit, dressing change kit, saline, syringe) and when the subject was released for IV therapy. The subject time in IR was based on the time the patient arrived in IR and when they were released for IV therapy.

Table 4 Procedural time and nurse time (minutes)

\begin{tabular}{|c|c|c|c|c|c|c|c|}
\hline \multirow[t]{3}{*}{ Time measurement } & \multicolumn{6}{|c|}{ Study arm } & \multirow[t]{3}{*}{$p$-Value } \\
\hline & \multicolumn{3}{|c|}{ Sherlock 3CG ${ }^{\mathrm{TM}}$ TCS } & \multicolumn{3}{|c|}{$\mathbf{B I} / \mathbf{C X R}$} & \\
\hline & Mean & SD & $\mathbf{n}$ & Mean & SD & $\mathbf{n}$ & \\
\hline Time from initiation of procedure to release for IV therapy & 33.93 & 25.63 & 60 & 176.32 & 249.84 & 60 & 0.001 \\
\hline Time associated with initial PICC placement & 33.93 & 25.63 & 60 & 29.05 & 7.84 & 60 & $\mathrm{n} / \mathrm{a}$ \\
\hline Time associated with confirming tip after initial placement & 0 & 0 & 0 & 78.04 & 51.67 & 60 & $\mathrm{n} / \mathrm{a}$ \\
\hline Time associated with additional procedures to confirm tip & 0 & 0 & 0 & 69.23 & 250.01 & 60 & $\mathrm{n} / \mathrm{a}$ \\
\hline PICC nurse time associated with initial PICC insertion & 42.00 & 16.35 & 60 & 42.50 & 16.81 & 60 & $\mathrm{n} / \mathrm{a}$ \\
\hline PICC nurse time associated with malposition adjustment after initial insertion & 0 & 0 & 0 & $1 \mathrm{I} .67$ & 7.97 & 7 & $\mathrm{n} / \mathrm{a}$ \\
\hline Subject time in IR & 0 & 0 & 0 & 100.40 & 107.06 & 6 & $\mathrm{n} / \mathrm{a}$ \\
\hline
\end{tabular}

Note: Only "Time from initiation of procedure to release for IV therapy" was tested statistically.

Abbreviations: TCS, Tip Confirmation System; BI/CXR, blind insertion with confirmatory chest X-ray; SD, standard deviation; IV, intravenous; PICC, peripherally inserted central catheter; n/a, not applicable; IR, Interventional Radiology. 
Two subjects (3\%) in the Sherlock 3CG TCS arm received a CXR after the initial PICC insertion (Table 5). While neither subject had a malposition, both were required to have a CXR before the subject could be released for IV therapy based on hospital protocol. All 60 subjects (100\%) in the BI/CXR arm received a CXR. Thirteen (22\%) received a second CXR after the initial CXR revealed an incorrect PICC tip location. Two (3\%) required a third CXR. The Wilcoxon rank sum test indicated that the Sherlock 3CG TCS has a statistically significant different mean number of total CXRs performed per subject than BI/CXR with the Sherlock 3CG TCS arm requiring fewer CXRs than the BI/CXR arm $(p<0.001)$.

No subjects in the Sherlock 3CG TCS arm had a malposition; 12 (20\%) subjects in the BI/CXR arm had a malposition during the initial PICC insertion (Table 6). Subsequently, two subjects had a remaining malposition following the first malposition adjustment attempt. Thus, 12 subjects had 14 recorded malpositions in the BI/CXR arm. The two-sample test of proportions indicated that the Sherlock 3CG TCS has a statistically significant different proportion of malpositions than BI/CXR with the proportion of malpositions for the Sherlock 3CG TCS arm being less than the proportion of malpositions for the BI/CXR arm $(p<0.001)$.

Additional secondary end points are shown in Table 7. These end points were tested statistically as part of a post hoc exploratory analysis.

Providers completed a survey following the procedure. They were asked about their satisfaction with the procedure

Table 5 CXRs performed

\begin{tabular}{|c|c|c|c|}
\hline \multirow[t]{2}{*}{ Attribute } & \multicolumn{2}{|l|}{ Study arm } & \multirow[t]{2}{*}{$p$-Value } \\
\hline & $\begin{array}{l}\text { Sherlock 3CG TM } \\
\text { TCS }(n=60)\end{array}$ & $\begin{array}{l}\text { BI/CXR } \\
(n=60)\end{array}$ & \\
\hline $\begin{array}{l}\text { Number of subjects receiving } \\
\text { a confirmatory CXR for } \\
\text { initial PICC placement }\end{array}$ & $2(3 \%)$ & $60(100 \%)$ & $\mathrm{n} / \mathrm{a}$ \\
\hline $\begin{array}{l}\text { Number of subjects receiving } \\
\text { a second CXR after first } \\
\text { malposition adjustment }\end{array}$ & $0(0 \%)$ & $13(22 \%)$ & $\mathrm{n} / \mathrm{a}$ \\
\hline $\begin{array}{l}\text { Number of subjects receiving } \\
\text { a third CXR after second } \\
\text { malposition adjustment }\end{array}$ & $0(0 \%)$ & $2(3 \%)$ & $\mathrm{n} / \mathrm{a}$ \\
\hline $\begin{array}{l}\text { Number of subjects who did } \\
\text { not receive a CXR }\end{array}$ & $58(97 \%)$ & $0(0 \%)$ & $\mathrm{n} / \mathrm{a}$ \\
\hline Total number of CXRs & 2 & 75 & $\mathrm{n} / \mathrm{a}$ \\
\hline $\begin{array}{l}\text { Mean number of total CXRs } \\
\text { performed per subject (SD) }\end{array}$ & $0.03(0.18)$ & $\mathrm{I} .25(0.5 \mathrm{I})$ & $<0.001$ \\
\hline
\end{tabular}

Note: Only "Mean number of total CXRs performed per subject (SD)" was tested statistically.

Abbreviations: CXRs, chest X-rays; TCS, Tip Confirmation System; BI/CXR, blind insertion with confirmatory chest X-ray; PICC, peripherally inserted central catheter; n/a, not applicable; SD, standard deviation.
Table 6 Number of subjects with malposition

\begin{tabular}{llll}
\hline Attribute & Study arm & p-Value \\
\cline { 2 - 3 } & $\begin{array}{l}\text { Sherlock 3CG TM } \\
\text { TCS }(\mathbf{n = 6 0 )}\end{array}$ & $\begin{array}{l}\text { BI/CXR } \\
(\mathbf{n}=60)\end{array}$ & \\
\hline $\begin{array}{l}\text { Number of subjects with } \\
\text { malposition during initial }\end{array}$ & $0(0 \%)$ & $12(20 \%)$ & $<0.00 \mathrm{I}$ \\
$\begin{array}{l}\text { PICC insertion } \\
\text { Number of subjects with } \\
\text { remaining malposition during }\end{array}$ & $0(0 \%)$ & $2(3 \%)$ & $\mathrm{n} / \mathrm{a}$ \\
$\begin{array}{l}\text { first malposition adjustment } \\
\text { Number of subjects with no } \\
\text { malposition }\end{array}$ & $60(100 \%)$ & $48(80 \%)$ & $\mathrm{n} / \mathrm{a}$ \\
\hline
\end{tabular}

Note: Only "Number of subjects with malposition during initial PICC insertion" was tested statistically.

Abbreviations: TCS, Tip Confirmation System; BI/CXR, blind insertion with confirmatory chest X-ray; PICC, peripherally inserted central catheter; n/a, not applicable.

Table 7 Other secondary end points

\begin{tabular}{|c|c|c|c|}
\hline \multirow[t]{2}{*}{ Attribute } & \multicolumn{2}{|l|}{ Study arm } & \multirow[t]{2}{*}{$p$-Value } \\
\hline & $\begin{array}{l}\text { Sherlock 3CG TM } \\
\text { TCS }(n=60)\end{array}$ & $\begin{array}{l}\text { BI/CXR } \\
(n=60)\end{array}$ & \\
\hline $\begin{array}{l}\text { Satisfaction with procedure } \\
\text { (overall) }\end{array}$ & $9.22(1.55)$ & $6.22(2.90)$ & $<0.001$ \\
\hline $\begin{array}{l}\text { Mean number of additional } \\
\text { venous access devices } \\
\text { required per subject }\end{array}$ & $0(0)$ & $0(0)$ & $\mathrm{n} / \mathrm{a}$ \\
\hline $\begin{array}{l}\text { Mean number of missed } \\
\text { medication doses per subject }\end{array}$ & $0(0)$ & $0.07(0.3 \mathrm{I})$ & 0.0807 \\
\hline $\begin{array}{l}\text { Mean number of missed } \\
\text { laboratory draws per subject }\end{array}$ & $0(0)$ & $0.07(0.25)$ & 0.0445 \\
\hline $\begin{array}{l}\text { Mean number of overtime } \\
\text { hours per subject }\end{array}$ & $0(0)$ & $0.07(0.25)$ & 0.0445 \\
\hline $\begin{array}{l}\text { Mean total procedural cost } \\
\text { (materials, labor, X-ray) }\end{array}$ & $\$ 273(\$ 67.03)$ & $\begin{array}{l}\$ 367 \\
(\$ 324.21)\end{array}$ & $<0.001$ \\
\hline
\end{tabular}

Notes: Mean (SD) shown. "Mean number of additional venous access devices required per subject" not tested statistically. All costs are reported in USD. Cost calculated as follows: mean (sum of material cost per PICC insertion, X-ray cost per PICC insertion, non-IR labor cost per PICC insertion, IR labor cost per PICC insertion).

Abbreviations: TCS, Tip Confirmation System; BI/CXR, blind insertion with confirmatory chest X-ray; n/a, not applicable; SD, standard deviation; PICC, peripherally inserted central catheter; IR, Interventional Radiology.

(overall), their satisfaction with the time to release the subject for IV therapy, and the perceived difficulty of completing the procedure. Each of these was asked on a scale from 0 to 10 with 0 meaning "not at all satisfied" or "not at all difficult" and 10 meaning "extremely satisfied" or "extremely difficult." Satisfaction with the procedure was statistically significantly different between the two arms $(p<0.001)$, with greater satisfaction in the Sherlock 3CG TCS arm. Providers in the Sherlock 3CG TCS arm were less likely to perceive the procedure as difficult to complete compared to the $\mathrm{BI} / \mathrm{CXR}$ arm.

No additional venous access devices were required due to a PICC not being released for IV therapy. The difference 
in the mean number of missed medication doses per subject was not different between arms ( 0 vs. 0.07$)$; however, the difference in the mean number of missed laboratory draws per subject was significantly different ( $p 0.0445 ; 0$ vs. 0.07 ).

Four subjects had a nurse work overtime to complete the PICC insertion. This totaled 4 hours, all recorded in the BI/ CXR arm. The difference in the mean number of overtime hours worked was significantly different ( $p$ 0.0445).

The costs associated with subjects undergoing a PICC insertion in the Sherlock 3CG TCS arm were statistically significantly different than those in the BI/CXR arm $(p<$ $0.001)$ with Sherlock 3 CG TCS costing less per insertion $(\mu$ \$273) than the BI/CXR arm ( $\mu$ \$367).

\section{Modeled economic results}

Clinical and cost outcomes were projected for Sherlock 3CG TCS compared with BI/CXR. The resource and cost inputs utilized in the modeled analysis are presented in Tables 8 and 9 , respectively.

Table 8 Resource data inputs utilized in the modeled analysis

\begin{tabular}{lll}
\hline Resource inputs & \multicolumn{2}{l}{ Study arm } \\
\cline { 2 - 3 } & $\begin{array}{l}\text { Sherlock 3CG TM } \\
\text { TCS }(\mathbf{n}=60)\end{array}$ & $\begin{array}{l}\text { BI/CXR } \\
(\mathbf{n}=60)\end{array}$ \\
\hline Initial CXR (\% patients) & $7 \%^{\mathrm{a}}$ & $\begin{array}{l}100 \% \\
(60 / 60)\end{array}$ \\
$\begin{array}{lll}\text { Additional CXR after malposition } \\
\text { (\% patients) }\end{array}$ & $0 \%(0 / 60)$ & $23 \%(14 / 60)$ \\
$\begin{array}{l}\text { Additional CXR but no adjustment } \\
\text { (\% patients) }\end{array}$ & $3 \%(2 / 60)$ & $2 \%(1 / 60)$ \\
$\begin{array}{l}\text { Procedures to correct malposition } \\
\text { (\% malpositions) }\end{array}$ & & \\
$\quad \begin{array}{l}\text { Bedside PICC team to correct } \\
\text { malposition }\end{array}$ & - & $50 \%(7 / 14)$ \\
$\begin{array}{l}\text { IR to correct malposition } \\
\text { No adjustment/procedure required } \\
\text { to correct malposition }\end{array}$ & - & $43 \%(6 / 14)$ \\
$\begin{array}{l}\text { Bedside staff time for malposition } \\
\text { adjustment (minutes) }\end{array}$ & - & $7 \%(1 / 14)$ \\
\hline
\end{tabular}

Notes: Resource inputs are derived from the Time and Motion study unless otherwise specified. a Given a range of patients that may be ineligible for ECG-based tip confirmation $\left(2.8 \%^{30}\right.$ to $\left.7 \%{ }^{\prime \prime}\right)$, it was conservatively assumed that $100 \%$ would attempt the Sherlock 3CG TCS and only $93 \%$ would be eligible. Patients may be ineligible for Sherlock 3CG TCS based on the reported proportion of patients who meet the exclusion criteria, such as patients with a non-discernible P-wave (eg, patients with atrial fibrillation, atrial flutter, severe tachycardia, or pacemaker-driven rhythm). ${ }^{\text {bT }}$ wo subjects (3\%) in the Sherlock 3 CG TCS arm received a CXR after the initial PICC placement. These subjects had implantable devices near the PICC line. The hospital's protocol required a CXR before the subjects could be released for IV therapy to confirm there was no disruption to the implantable device due to the PICC line. One subject (2\%) in the BI/CXR arm received a second CXR after the initial PICC placement despite not having a malposition. A second CXR was ordered to better visualize the PICC tip location.

Abbreviations: TCS, Tip Confirmation System; BI/CXR, blind insertion with confirmatory chest X-ray; CXR, chest X-ray; PICC, peripherally inserted central catheter; IR, Interventional Radiology; ECG, electrocardiogram; IV, intravenous.
Table 9 Cost inputs utilized in the modeled analysis

\begin{tabular}{ll}
\hline Cost inputs & USD \\
\hline Average upcharge cost per PICC for Sherlock 3CG TM & $\$ 87$ \\
TCS vs. BI/CXR & \\
Capital equipment cost & \\
BI/CXR & $\$ 14,000^{\mathrm{a}}$ \\
$\quad$ Sherlock 3CG TCS & $\$ 18,300^{\mathrm{a}}$ \\
CXR cost & $\$ 60.80^{\mathrm{b}}$ \\
Material costs to correct malposition at bedside & $\$ 31.32$ \\
Bedside staff hourly wage & $\$ 34.30$ \\
Cost of procedures requiring IR to correct malpositions & $\$ 862.50^{\mathrm{c}}$ \\
\hline
\end{tabular}

Notes: Cost inputs are derived from the Time and Motion study unless otherwise specified. a Capital costs for BI/CXR were based on an approximate cost of $\$ 14,000$ for ultrasound, and capital costs for Sherlock 3CG TCS were based on $\$ 14,000$ for Bard Site Rite $6^{\mathrm{TM}}$ ultrasound plus an approximate $\$ 4,300$ upcharge for the Sherlock 3CG TCS capital equipment upgrade. The capital equipment life cycle was assumed to be 5 years, and therefore, only one unit would be required within the 3-year time horizon of the cost analysis. ${ }^{31}$ TThe cost of a CXR was based on the 2016 national average payment rate for a CXR (I frontal view, CPT 7|0I0). ${ }^{32}$ cThe cost of procedures requiring IR to correct malpositions was based on the 2016 national average payment rate for repositioning of venous catheter (CPT 36597). ${ }^{32}$

Abbreviations: TCS, Tip Confirmation System; BI/CXR, blind insertion with confirmatory chest X-ray; CXR, chest X-ray; IR, Interventional Radiology.

For a hypothetical population of 1,000 annual patients, adoption of Sherlock 3CG TCS was predicted to be a costsaving strategy compared with BI/CXR for all 3 years in the analysis (Table 10). The cumulative cost savings over 3 years was $\$ 215,899$ (Figure 2).

When projecting clinical outcomes, compared with $\mathrm{BI} /$ CXR, the adoption of Sherlock 3CG TCS was predicted to avert 1,147 CXRs annually, averting almost 3,500 CXRs in 3 years. In addition, nurse overtime was predicted to be reduced by 70 hours annually, for a 3 -year cumulative savings of 210 hours of nurse overtime. Finally, the 3 -year cumulative patient wait time for radiology malposition correction was predicted to be reduced by 502 hours (167 hours annually) when switching from BI/CXR to Sherlock 3CG TCS.

One-way sensitivity analysis of resource and cost inputs demonstrated that the 3-year cumulative change in cost results was robust to a wide range in parameter assumptions. The model results were most affected by variation in the proportion of BI/CXR patients requiring additional CXRs after malposition, the average upcharge cost for Sherlock 3CG TCS vs. BI/CXR, and the proportion of malpositions cor-

Table 10 Total cost per year based on 1,000 annual PICC placements

\begin{tabular}{lllll}
\hline $\begin{array}{l}\text { Year of } \\
\text { analysis }\end{array}$ & $\begin{array}{l}\text { Total } \\
\text { cost with } \\
\text { BI/CXR }\end{array}$ & $\begin{array}{l}\text { Total cost } \\
\text { with Sherlock } \\
\text { 3CG }\end{array}$ TM TCS & $\begin{array}{l}\text { Incremental } \\
\text { difference }\end{array}$ & \\
\hline Year I & $\$ 320,682$ & $\$ 251,583$ & $-\$ 69,100$ & Cost savings \\
Year 2 & $\$ 306,682$ & $\$ 233,283$ & $-\$ 73,400$ & Cost savings \\
Year 3 & $\$ 306,682$ & $\$ 233,283$ & $-\$ 73,400$ & Cost savings \\
\hline
\end{tabular}

Abbreviations: PICC, peripherally inserted central catheter; $\mathrm{BI} / \mathrm{CXR}$, blind insertion with confirmatory chest X-ray; TCS, Tip Confirmation System. 


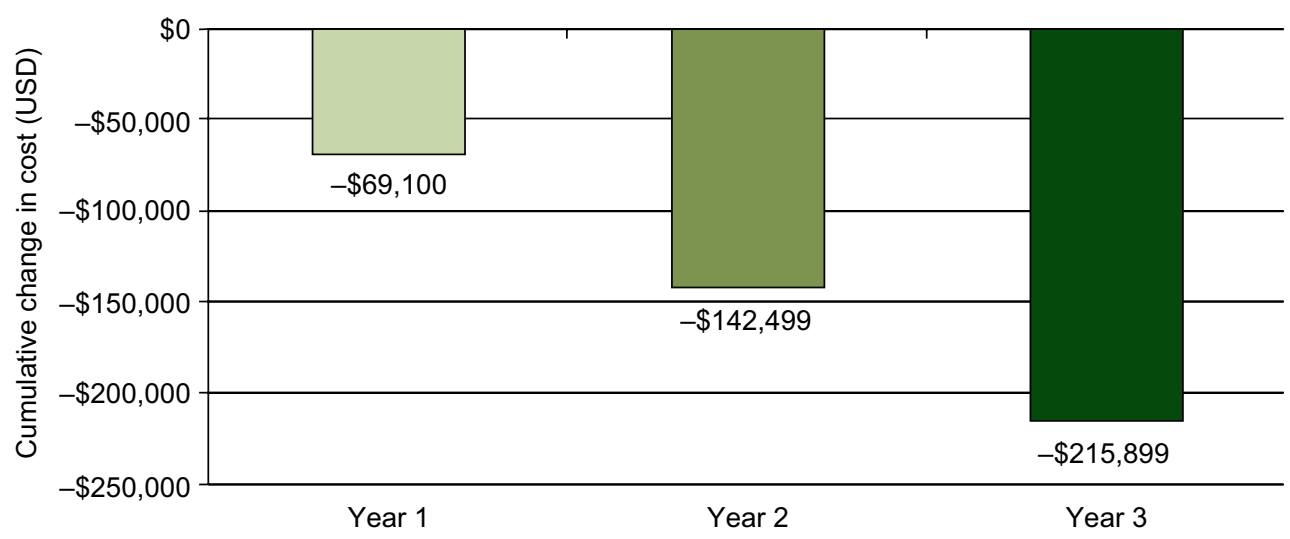

Figure 2 Cumulative change in total cost by year (in USD).

Model input varied $\pm \mathbf{2 0} \%$ (lower estimate, upper estimate) BI/CXR patients requiring an additional chest X-ray after malpositions (19\%, 28\%) Average upcharge cost per PICC for Sherlock 3CG ${ }^{\text {TM }}$ TCS vs. BI/CXR $(\$ 69.60, \$ 104.40)$ Malpositions corrected in the IR $(34 \%, 51 \%)$ Cost of procedures requiring IR to correct malpositions $(\$ 420.80, \$ 631.20)$ Number of PICCs placed annually $(800,1,200)$ Chest X-ray cost $(\$ 48.64, \$ 72.96)$ Capital equipment cost for Sherlock 3CG ${ }^{\text {TM }}$ TCS $(\$ 14,640, \$ 21,960)$ Capital equipment cost for BI/CXR $(\$ 11,200, \$ 16,800)$ Malpositions corrected at bedside $(40 \%, 60 \%)$ Sherlock 3 CG $^{\mathrm{TM}}$ TCS patients requiring initial chest X-ray $(6 \%, 8 \%)$

Material cost to correct malposition at bedside (\$25.06, \$37.58) Sherlock $3 \mathrm{CG}^{\mathrm{TM}}$ TCS patients requiring chest $\mathrm{X}$-ray but no adjustment $(2.7 \%, 4.0 \%)$ $\mathrm{BI} / \mathrm{CXR}$ patients requiring chest $\mathrm{X}$-ray but no adjustment (1.3\%, 2.0\%) Bedside nurse wage $(\$ 27.44, \$ 41.16)$

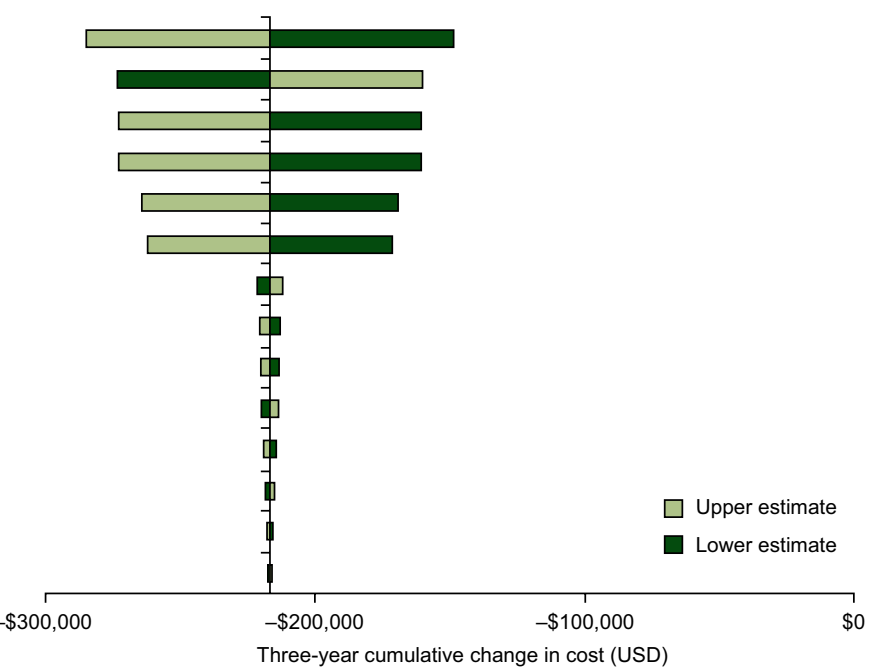

Figure 3 One-way sensitivity analysis on the 3-year cumulative change in cost.

Abbreviations: BI/CXR, blind insertion with confirmatory chest X-ray; PICC, peripherally inserted central catheter; TCS, Tip Confirmation System; IR, Interventional Radiology.

rected in IR. The tornado diagram demonstrates the one-way sensitivity analysis on the 3-year cumulative change in cost (Figure 3) and demonstrates the impact of each parameter on the model results. Parameters are presented in Figure 3 in descending order from the greatest to the least impactful.

\section{Discussion}

This study demonstrates that the Sherlock 3CG TCS has several advantages compared with BI/CXR.

The primary end point of this study was to evaluate a time difference between technologies. Clinicians using the Sherlock 3CG TCS started the insertion procedure and released subjects for IV therapy substantially and significantly faster than with BI/CXR. The mean Sherlock 3CG TCS case took 34 minutes to release a subject for IV therapy, compared with a mean time of 176 minutes for the $\mathrm{BI} / \mathrm{CXR} \mathrm{arm}$. The mean difference was $>2$ hours (142 minutes) $(p<0.001)$. By removing the reliance on the CXR tip confirmation process and reducing the occurrence of malpositions with real-time navigation during insertion and immediate tip location confirmation, providers at Sherlock 3CG TCS hospitals were able to deliver therapeutic medications earlier.

No malpositions were recorded for PICC insertions using the Sherlock 3CG TCS, while $20 \%$ of subjects in the BI/ CXR arm had a malposition $(p<0.001)$. When malpositions occurred, the majority were corrected with additional, but minimal, procedural time (mean 11.67 minutes). Hospitals using Sherlock 3CG TCS eliminated the need to use resources to correct these malpositions (eg, additional correction procedures performed at bedside or in IR, follow-up CXRs).

It is possible that the time required to correct malpositions was recorded in ideal conditions (based on the hospitals that participated in this study). The IR services at the two BI/CXR hospitals were mature and had the bandwidth to provide corrective services in an expedited fashion. This may not be possible in smaller hospitals, and thus, the total time 
to correct malpositions may be underestimated in hospitals with less robust IR backup.

On average, subjects in the Sherlock 3CG TCS arm received $0.03 \mathrm{CXRs}$, compared with 1.25 in the $\mathrm{BI} / \mathrm{CXR}$ arm. Significantly fewer CXRs were required in the Sherlock 3 CG TCS arm $(p<0.001)$.

From a hospital perspective, the cost analysis predicted that Sherlock 3CG TCS adoption would result in cost savings when compared with BI/CXR for a hypothetical population of 1,000 patients admitted annually over 3 years. The cumulative 3-year savings were projected to be $\$ 215,899$ for this population. Overall, the reduction in downstream resource utilization associated with Sherlock 3CG TCS not only offset its initial acquisition costs but also led to cost savings when compared with BI/CXR. Given the growing requirement for PICC insertion and the era of cost containment, the predicted savings from the cost analysis are an important consideration for hospital decision-makers.

The cost analysis was primarily based on the data obtained from this Time and Motion study, and the results are consistent with the findings of other published economic evaluations for Sherlock 3CG TCS. In 2015, the National Institute for Health and Care Excellence (NICE) recommended the adoption of Sherlock 3CG TCS in the UK based on clinical evidence and UK-modeled health economic benefits. ${ }^{33}$ In 2016, Dale et $\mathrm{al}^{34}$ discussed the NICE submission and indicated that the use of Sherlock 3 CG TCS may be approximately cost neutral compared to $\mathrm{BI} / \mathrm{CXR}$. It is important to note that the authors went on to postulate that there may be cost savings relevant to health care decision-makers if there were reductions in nurse time, X-ray provision, portering (patient transfer), or the number of reinsertions required. The Time and Motion results presented here and the cost analysis conducted support the comments made by Dale et al.

The clinical and cost outcomes of Sherlock 3CG TCS demonstrated by the Time and Motion study and projected in the cost analysis are aligned with US health care reform, which promotes resource efficiencies, high-quality care, and patient and health care worker satisfaction. For example, given the risk associated with radiation exposure from X-rays, ${ }^{35,36}$ there are potential safety benefits for patients and health care workers if CXRs can be averted. In addition, based on the results of the Time and Motion study, there were no malpositions associated with Sherlock 3CG TCS when compared with BI/CXR and therefore resulted in substantially less material and fewer personnel required to correct malpositions. Finally, hospital personnel may have additional time savings when utilizing Sherlock 3CG TCS, and therefore, they may have additional time for other activities, promoting efficiencies in the hospital work environment. Taken together, these resource savings can translate into improved workflow and reduced hospital costs.

Within the study, there were a mix of PICC teams across the sites, and there were often two health care personnel present at a given time. The impact of reduced resource use on total cost will vary by institution, based on the number of health care personnel. In the cost analysis, the average nursing time associated with the correction of malposition at bedside was included in the cost projections. However, the Time and Motion study also demonstrated significant differences in the mean number of nursing overtime hours. Although not included in the cost analysis, the projected 3 -year cumulative savings in nursing overtime associated with Sherlock 3CG TCS was 210 hours. When coupled with the predictable reduction in the reliance on CXRs and reduction in malpositions, there are substantial benefits for consideration by health care decision-makers.

There are some limitations to consider for this study. The Time and Motion study data were collected at four hospitals, which were not randomized for inclusion. As such, their patient populations and procedures may not represent all hospitals in the US. Further, rather than randomly assigning study arm at the site, we created a treatment cohort, whose selection approach was similar at each site. Any and all patients requiring PICC insertion from a specific time point until study completion were screened for inclusion and exclusion; however, as the study did not employ a matched design, subjects enrolled in each arm may not be equivalent in terms of physiologic factors, which could have produced a spurious relationship between study outcomes and the technologies assessed. Additionally, procedural differences (administrative, communication, other) between the study arms may have led to differences in results, beyond insertion technology. While the abovementioned circumstances could have contributed to the results, the fact that malposition rates varied so much between the arms suggest that nonadministrative factors account for much of the time differences observed. Care was taken to ensure the enrolled sites were comparable in PICC insertion volume. An initial Site Feasibility Questionnaire (SFQ) was sent and collected from prospective sites to help ensure consistency across sites. Care was also taken to confirm data were collected as similarly as possible across sites, through training and site monitoring. Similar care was taken with subject enrollment, in that study protocol was followed strictly. Although the clinicians who participated in this study were instructed to work "normally" 
when inserting PICCs, having a study observer on-site may have affected behavior. While the effect of being observed has been previously recognized (Hawthorne effect) as a potentially confounding influence, the study observers and data collection process were minimally intrusive during the overall process and any effect is likely negligible. Further, any varied behavioral effect of being observed would have occurred in both arms.

In addition, there are some limitations associated with the cost analysis. The time study was not powered to detect differences in resource outcomes, as they were not primary end points. However, health economic good research practices acknowledge studies that are underpowered for economic outcomes and consider them acceptable data sources for modeled analyses. ${ }^{37}$ Furthermore, the study data used to inform the cost analysis were obtained from relatively small patient numbers in the Time and Motion study. The results of the analysis may be strengthened by additional data from even larger studies or using other real-world cost data. However, it is important to note that the Time and Motion study was powered to meet the primary end point, and the sample size was in the range of other medical device studies. Finally, although the model attempted to include a comprehensive range of relevant parameters, not all possible variables were included. Specifically, when changing over from one technology to another, there are upfront capital costs and costs associated with staff training required to avoid potential practical problems that may arise as staff begin using a new technology. This modeled analysis included capital costs but did not include training costs given that the staff using Sherlock 3CG TCS at participating sites were already trained and additional costing data for training were not collected. However, based on similar models that evaluated the switch between technologies, nurse training costs were among the least impactful parameters from both US and non-US perspectives. ${ }^{38,39}$ Therefore, the exclusion of training costs in this modeled analysis is not expected to impact the conclusions.

Despite these limitations, this study used an a priori protocol to gather real-world evidence to evaluate differences between the Sherlock $3 \mathrm{CG}$ TCS and BI/CXR. A robust sample, data collection techniques (including pilot testing), and best practices related to analysis and health economic modeling ensured that these findings will contribute greatly to hospital decision making across the US.

\section{Conclusion}

The results from this study demonstrate the Sherlock $3 \mathrm{CG}$ TCS to be superior to $\mathrm{BI} / \mathrm{CXR}$ with regard to time to release patient for IV therapy, malposition rates, and hospital costs.
The Sherlock 3CG TCS has the potential to increase PICC bedside insertion productivity and reduce hospital resource utilization by reducing insertion time and reducing the rate of malpositions.

This study and the cost analysis demonstrate superiority using real-world evidence and a cost analysis designed to generalize the results. While it may not be used for every patient (given its indications for use), the Sherlock 3CG TCS has proven superior in its appropriate use cases. Combined, these findings provide a strong case for hospitals to consider adopting the Sherlock 3CG TCS as an alternative to CXR confirmation.

\section{Acknowledgments}

The financial support for this study was provided by C. R. Bard, Inc. C. R. Bard participated in protocol creation, enrollment monitoring, data analysis, interpretation of data, review, and approval of the manuscript. This study was financially supported by C. R. Bard, Inc.

\section{Author contributions}

All the authors have contributed substantially to the conception and design of the study and the acquisition, analysis, and interpretation of data. They have contributed in drafting the manuscript and revision for the final approval.

\section{Disclosure}

Dr Tomaszewski, Ms Ferko, and Ms Hollmann are employees of organizations under contract with C.R. Bard to develop and execute this study. The authors report no other conflicts of interest in this work.

\section{References}

1. Moureau N, Gabriel J. Peripherally inserted central catheters. In: Hamilton H, Bodenham A, editors. Central Venous Catheters. Chichester, England: Wiley-Blackwell; 2009:114-126.

2. Infusion Nurses Society. Infusion nursing standards of practice. $J$ Infusion Nurs. 2016;39(Number 1S):S47. Standard 23, Practice Criteria C.

3. National Association of Vascular Access Networks. Position paper: tip location of peripherally inserted central catheters. JVasc Access Devices. 1998;3:1-4.

4. Dariushnia SR, Wallace MJ, Siddiqi NH, et al. Quality improvement guidelines for central venous access. J Vasc Interv Radiol. 2010;21(7): 976-981.

5. Oncology Nurses Society. Access Device Guidelines: Recommendations for Nursing Practice and Education. 3rd ed. Pittsburgh, PA: Oncology Nurses Society; 2010.

6. Cardella JF, Fox PS, Lawler JB. Interventional radiologic placement of peripherally inserted central catheters. J Vasc Interv Radiol. 1993; 4(5):653-660.

7. Trerotola SO, Thompson S, Chittams J, Vierregger KS. Analysis of tip malposition and correction in peripherally inserted central catheters placed at bedside by a dedicated nursing team. JVasc Interv Radiol. 2007; 18(4):513-518.

8. McGee DC, Gould MK. Preventing complications of central venous catheterization. N Engl J Med. 2003;348(12):1123-1133. 
9. Amerasekera SS, Jones CM, Patel R, Cleasby MJ. Imaging of the complications of peripherally inserted central venous catheters. Clin Radiol. 2009;64(8):832-840.

10. Smith B, Neuharth RM, Hendrix MA, McDonnall D, Michaels AD. Intravenous electrocardiographic guidance for placement of peripherally inserted central catheters. J Electrocardiol. 2010;43(3): 274-278.

11. Pittiruti M, La Greca A, Scoppettuolo G. The electrocardiographic method for positioning the tip of central venous catheters. JVasc Access. 2011;12(4):280-291.

12. Jin J, Chen C, Zhao R, Li A, Shentu Y, Jiang N. Repositioning techniques of malpositioned peripherally inserted central catheters. J Clin Nurs. 2013;22(13-14):1791-1804.

13. Song L, Li H. Malposition of peripherally inserted central catheter: experience from 3,012 patients with cancer. Exp Ther Med. 2013;6(4): 891-893.

14. Oliver G, Jones M. ECG or X-ray as the 'gold standard' for establishing PICC-tip location? Br J Nurs. 2014;23(Suppl 19):S10-S16.

15. Venkatesan T, Sen N, Korula PJ, et al. Blind placements of peripherally inserted antecubital central catheters: initial catheter tip position in relation to carina. Br J Anaesth. 2007;98(1):83-88.

16. Caers J, Fontaine C, Vinh-Hung V, et al. Catheter tip position as a risk factor for thrombosis associated with the use of subcutaneous infusion ports. Support Care Cancer. 2005;13(5):325-331.

17. Lee AY, Kamphuisen PW. Epidemiology and prevention of catheterrelated thrombosis in patients with cancer. JThromb Haemost. 2012;10(8): 1491-1499.

18. Orme RM, McSwiney MM, Chamberlain-Webber RF. Fatal cardiac tamponade as a result of a peripherally inserted central venous catheter: a case report and review of the literature. Br J Anaesth. 2007;99(3):384-388.

19. Petersen J, Delaney JH, Brakstad MT, Rowbotham RK, Bagley CM Jr. Silicone venous access devices positioned with their tips high in the superior vena cava are more likely to malfunction. Am J Surg. 1999 178(1):38-41.

20. Robinson MK, Mogensen KM, Grudinskas GF, Kohler S, Jacobs DO. Improved care and reduced costs for patients requiring peripherally inserted central catheters: the role of bedside ultrasound and a dedicated team. JPEN J Parenter Enteral Nutr. 2005;29(5): 374-379.

21. Sainathan S, Hempstead M, Andaz S. A single institution experience of seven hundred consecutively placed peripherally inserted central venous catheters. J Vasc Access. 2014;15(6):498-502.

22. Hockley SJ, Hamilton V, Young RJ, et al. Efficacy of the CathRite system to guide bedside placement of peripherally inserted central venous catheters in critically ill patients: a pilot study. Crit Care Resusc. 2007;9(3):251-255.

23. Naylor CL. Reduction of malposition in peripherally inserted central catheters with tip location system. J Assoc Vasc Access. 2007;12(1): 29-31.

24. Meyer BM. Implementing and maintaining an infusion alliance. J Infus Nurs. 2010;33(5):292-303.

25. Bard Access Systems. Sherlock $3 C G^{\mathrm{TM}}$ Tip Confirmation: Standalone Instructions for Use. Salt Lake City, UT: Bard Access Systems; 2013.
26. Johnston AJ, Holder A, Bishop SM, See TC, Streater CT. Evaluation of the Sherlock 3CG Tip Confirmation System on peripherally inserted central catheter malposition rates. Anaesthesia. 2014;69(12):1322-1330.

27. Lelkes V, Kumar A, Shukla PA, Contractor S, Rutan T. Analysis of the Sherlock II tip location system for inserting peripherally inserted central venous catheters. Clin Imaging. 2013;37(5):917-921.

28. Burke TA, McKee JR, Wilson HC, Donahue RM, Batenhorst AS, Pathak DS. A comparison of time-and-motion and self-reporting methods of work measurement. J Nurs Adm. 2000;30(3):118-125.

29. Zheng K, Guo MH, Hanauer DA. Using the time and motion method to study clinical work processes and workflow: methodological inconsistencies and a call for standardized research. J Am Med Inform Assoc. 2011;18(5):704-710.

30. Pittiruti M, Bertollo D, Briglia E, et al. The intracavitary ECG method for positioning the tip of central venous catheters: results of an Italian multicenter study. J Vasc Access. 2012;13(3):357-365.

31. Bard Access Systems. Data on File. Salt Lake City, UT: Bard Access Systems; 2016.

32. Centers for Medicare \& Medicaid Services [webpage on the Internet]. CMS-1633-FC; CMS-1607-F2. 2016 OPPS Final Rule Addenda: $B$ 10.26.15. 2016. Available from: https://www.cms.gov/Medicare/ Medicare-Fee-for-Service-Payment/HospitalOutpatientPPS/HospitalOutpatient-Regulations-and-Notices-Items/CMS-1633-FC.html. Accessed August 12, 2016.

33. National Institute for Health and Care Excellence. NICE Medical Technology Guidance [MTG24]: The Sherlock 3CG Tip Confirmation System for Placement of Peripherally Inserted Central Catheters. London: National Institute for Health and Care Excellence; 2015.

34. Dale M, Higgins A, Carolan-Rees G. Sherlock 3CG((R)) Tip Confirmation System for placement of peripherally inserted central catheters: a NICE Medical Technology Guidance. Appl Health Econ Health Policy. 2016;14(1):41-49.

35. Ju F, Gong X, Jiang L, et al. Chronic myeloid leukaemia following repeated exposure to chest radiography and computed tomography in a patient with pneumothorax: a case report and literature review. Oncol Lett. 2016;11(4):2398-2402.

36. U.S. Department of Health and Human Services: National Cancer Institute [webpage on the Internet]. Discovering the Causes of Cancer and the Means of Prevention (Research): U.S. Radiologic Technologists Cohort. 2016. Available from: http://dceg.cancer.gov/research/who-westudy/cohorts/us-radiologic-technologists. Accessed August 12, 2016

37. Ramsey SD, Willke RJ, Glick H, et al. Cost-effectiveness analysis alongside clinical trials II-An ISPOR Good Research Practices Task Force report. Value Health. 2015;18(2):161-172.

38. Pswarayi C, Kara R, Hollmann S, Ferko N, Dawson D, Delatore P. Modelled U.K. and U.S. Analyses Demonstrate Sherlock 3CG® Tip Confirmation System for peripherally inserted central catheter placement is associated with favourable health economic outcomes. Value Health. 2015;18(7):A369.

39. Dale M, Morgan H, Peirce S, et al. Cedar healthcare technology centre. External assessment centre report: Sherlock $3 \mathrm{CG}$ tip confirmation system for placement of peripherally inserted central catheters. Cardiff Vale University Health Board. 2014; Version 1.3 [updated 2014 Nov 4]:1-91. Available from: http://www.cedar.wales.nhs.uk/sherlock. Accessed August 12, 2016.

\section{ClinicoEconomics and Outcomes Research}

\section{Publish your work in this journal}

ClinicoEconomics and Outcomes Research is an international, peerreviewed open-access journal focusing on health technology assessment, pharmacoeconomics and outcomes research in the areas of diagnosis, medical devices, and clinical, surgical and pharmacological intervention. The economic impact of health policy and health systems organization also constitute important areas of coverage. The manuscript management system is completely online and includes a very quick and fair peer-review system, which is all easy to use. Visit http://www.dovepress.com/testimonials.php to read real quotes from published authors. 